\title{
The management of upper urinary tract calculi by piezoelectric extracorporeal shock wave lithotripsy in spinal cord injury patients
}

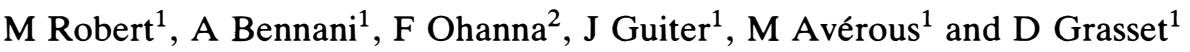 \\ ${ }^{1}$ Service d'Urologie, Hôpital Lapeyronie, 371 Avenue Doyen Giraud, 34295 Montpellier Cedex 5; ${ }^{2}$ Centre \\ Propara, 263 Rue du Caducée, Parc Euromédecine, 34195 Montpellier Cedex 5, France
}

From May 1988 to September 1994, 15 spinal cord injury patients were treated by piezoelectric extracorporeal shock wave lithotripsy. Aged from 23 to 71 years (mean $=39$ ), they presented with a total of 23 stones, of which 18 were located in the calyces, three in the renal pelvis and two in the proximal ureter. The maximum dimensions of calculi varied from 5 to $35 \mathrm{~mm}($ mean $=11)$. Patients were placed in a dorsal decubitus position during the sessions, three being sedated with diazepam, while the other 12 remained unsedated. All were treated routinely with systemic antibiotics. Auxiliary procedures consisted of two pyelocalyceal flushings, three double $\mathrm{J}$ ureteral stenting and three ureteroscopies with fragment removal with a Dormia basket. No episode of autonomic dysreflexia was observed. Short term side effects were limited to a few cases of gross haematuria which regressed spontaneously. Overall, eight successes (53\%), and seven failures $(47 \%)$, were registered. Of the failures, one was the result of a partial fragmentation, while six were related to intrarenal retention of residual fragments resulting in four cases in rapid recurrences. Extracorporeal shock wave lithotripsy can be easily applied to spinal cord injury patients. Its usefulness and limitations need to be well understood and a global consideration must be applied to the prevention and early detection of the upper urinary calculi in this exposed population of patients.

Keywords: spinal cord injury; urinary lithiasis; extracorporeal shock wave lithotripsy

\section{Introduction}

Extracorporeal shock waves lithotripsy (ESWL) has undergone a remarkable development during the last decade and has dramatically changed the urological management of upper urinary tract calculi, becoming its main modality. ${ }^{1-3}$ This pathology has high rates of ocurrence and recurrence among spinal cord injury patients, and has a particularly poor prognosis in this population. ${ }^{4-7}$ In this study, we have focused on the efficacy of extracorporeal lithotripsy in paraplegic and tetraplegic patients.

\section{Material and methods}

From May 1988 to September 1993, we treated 15 spinal cord injury patients by echo-guided piezoelectric ESWL (EDAP LT 01). These patients, of whom 13 were males and two females, were aged from 23 to 71 years $($ mean $=39)$ and presented with radio-opaque upper urinary tract calculi. Their spinal cord damage was secondary to closed trauma in 12 cases, to a bullet injury in two and to iatrogenic compression in one. The level of the spinal lesions was cervical in eight cases, thoracic in six and lumbar in one.

Seven patients had a previous history of urinary stone of whom two were treated prior to spinal cord injury by pyelo or uretero surgical lithotomy and five after traumatism by surgical (three) and percutaneous nephrolithotomies (two) or nephrectomy (one). Concerning modalities of micturition, two had spontaneous bladder voiding, six needed hypogastric percussion, six required self catheterisation (one enterocystoplasty according to the technique of Koch), and one had an indwelling urethral catheter. Moreover, 12 of these patients $(80 \%)$ had recurrent urinary tract infections, the principal organisms being Proteus, Pseudomonas and E. Coli.

The urinary calculi were discovered during systematic investigations in 13 cases while in two they were revealed by febrile episodes or autonomic dysreflexia. Stones were unilateral in 14 cases and bilateral in one. Of the 14 unilateral cases, seven patients had multiple calculi. In all, 23 calculi were found, of which 18 were located in the calyces, three in the renal pelvis and two in the proximal ureter, with maximum dimensions ranging from 5 to $35 \mathrm{~mm}($ mean $=11)$.

ESWL was performed in the dorsal decubitus position, without any sedation in 12 cases and under sedation with diazepam in three cases. Every patient received parenteral antibiotics immediately before ESWL and for 1 week when preoperative urine cultures were positive.

Two ureteral calculi which were not accessible to 
echographic detection were pushed back into the pyelocalyceal region by endoscopy prior to ESWL. In these two cases, a double $\mathrm{J}$ ureteral stent was implanted immediately after flushing. In another case involving the most voluminous calculus of this series, a ureteral endoprothesis was implanted before treatment so as to limit the risk of secondary ureteral obstruction due to migrating fragments.

\section{Results}

We carried out 63 ESWL sessions (EDAP LT 01) focused on 23 calculi, without any specific problems or significant secondary effects. There were no episodes of autonomic dysreflexia and in particular of hypertension during the sessions. Antibiotic prophylaxis avoided symptomatic urinary tract infections and no change in serum creatinine was detected after ESWL. The only side effect observed was a few cases of gross haematuria which spontaneously regressed without any further infectious or obstructive consequences.

The number of sessions per patient varied from one to seven $($ mean $=4.2)$. Auxiliary measures consisted of ureteroscopy in two cases for the removal with a Dormia basket, of fragments which were deemed not likely to be spontaneously evacuated.

From 1 to 3 months after the lithotripsy, follow-up examinations consisted of plain abdomen radiograph and ultrasonic evaluation. In cases of residual fragments above $3 \mathrm{~mm}$ in diameter, a second ESWL session was rapidly performed. Otherwise, follow-up evaluation was scheduled for 3 months later.

Overall, eight (53\%) treatment cases were successful with patients totally free of stone while seven $(47 \%)$ cases were failures.

The eight patients successfully treated had 11 calculi (seven in the calyces, two in the renal pelvis and two in the proximal ureter) with maximum dimensions ranging from 5 to $35 \mathrm{~mm}($ mean $=10.7)$. The number of sessions for patients of this group ranged from 1 to 3 (mean $=1.7$ ). Despite successful stone removal, five of the six patients of this group whose preoperative urine cultures were positive continued to have persistent bacteriuria. After a follow-up period of 9-12 months, two $(25 \%)$ of them had stone recurrence in the lower calyx despite an initial perfect result confirmed at 6 months.

The seven unsuccessfully treated patients had a total of 12 stones (11 in the calyces and one in the renal pelvis) with maximum dimensions ranging from 6 to $25 \mathrm{~mm}($ mean $=11.7)$. The number of sessions per patient varied from 2 to $7($ mean $=5.6)$. One failure was the result of marginal erosion of the calculus and six were related to calculi well fragmented but not totally eliminated. Four patients $(66 \%)$ had rapidly evolving and recurring calculi requiring multiple ESWL sessions, while the other two (33\%) showed a stable residual stone fragment $36-48$ months afterwards. Two patients of this group required subsequent percutaneous nephrolithotomy but five patients did not justify other than ESWL urological treatment.
Stones were morphologically similar in the two groups; however, the relative incidence of lower calyx stone was higher among the unsuccessful cases $(50 \%)$, compared with the sucessful cases $(18 \%)$.

\section{Discussion}

About $8 \%$ of spinal cord injury patients are afflicted by upper urinary tract stones, usually asymptomatic, mainly composed of struvite and carbonate apatite and associated with infection. Bilateral staghorn calculi and subsequent renal failure has an overall 10 -year mortality rate of $50 \%$, and is, thus, one of the major cases of deaths among these patients..$^{4,5,8}$

The major determining factors in this lithogenic process are hypercalciuria due to prolonged immobilisation, urinary stasis, and recurrent urinary tract infections. ${ }^{4-7,9}$ Stone development is often rapid and precocious and the majority of calculi appear within the first 2 years following the spinal cord injury. ${ }^{10}$

In the past, stones detected were essentially staghorn or partial staghorn calculi, unsuitable for ESWL treatment. Over a few years, the epidemiology of this pathology has been transformed by the use of systematic follow-up and by a better handling of lower urinary tract dysfunction induced by spinal cord damage. Decreases in the incidence of large renal stone have increased the interest in ESWL for spinal cord injury patients. ${ }^{10-15}$

Stone location determines the positioning of the patient in ESWL and thus dorsal decubitus is used for renal and proximal ureteral calculi and the prone position for medial and distal ureteral calculi. The dorsal decubitus position is normally readily performed, but the existence of hip joint limitations in spinal cord injured patients can render the prone position difficult.

In these subjects, and especially in tetraplegic patients, nociceptive stimuli can trigger episodes of autonomic dysreflexia, which are characterised by hypertension. General anaesthesia or systematic locoregional anaesthesia has been advocated in order to prevent these phenomena. Patients who are prone to such complications could benefit from local anaesthesia over the area where the shock waves will penetrate. Strict surveillance of blood pressure is warranted, as well as immediate treatment when hypertension appears. ${ }^{12-15}$

Our results are inferior to the usual results of ESWL of which the average success rate is in the order of $70-85 \%$ in cases of calculi measuring less than $15 \mathrm{~mm}$. The efficiency of this treatment depends not only on the stone size, but also on friability and topography. Di-hydroxide calcium oxalate or magnesiumammonium-phosphate calculi are particularly fragile, while lower calyx stones often present elimination problems. $^{1-3,16-22}$

Papers dealing specifically with ESWL of calculi among spinal injury patients are rare. In a series of 32 patients treated with a DORNIER HM 3, Lazare reports success rates in the order of $90 \%$ for calculi 
measuring less than $20 \mathrm{~mm}$, and $70 \%$ for those between 20 and $40 \mathrm{~mm}$. Neuwirth reports a $50 \%$ success rate with a series of eight patients, but here the calculi were much more voluminous. ${ }^{12-15}$ In studies dealing with lithiasis arising from infections, which occur at a high rate among spinal cord damaged patients, the success rate of ESWL is around $50 \%$. In addition, the persistence of urinary infections associated with residual fragments greater than $5 \mathrm{~mm}$ is synonymous with a rapid recurrence in $80 \%$ of cases. A prolonged antibiotic treatment for 6-9 months permits complete eradication of the infection in $85 \%$ of cases. ${ }^{23-25}$ Failure to eradicate infection or stagnation of fragments in the lower calyx may explain most of the rapid recurrences as well as the repeated sessions of ESWL which occurred in our experience.

Poor ultrasonic localisation of calculi was another cause of failure in our experience, whether due to the small size of some calculi, or to the presence of numerous ultrasound artefacts resulting from previous renal surgery. On the other hand, it is obvious that the piezoelectric extracorporeal shock waves had not been sufficiently powerful when some large stones were concerned.

The therapeutic performance of ESWL benefits from regular improvements. Technical advances in the fields of fragmentation power and detection are particularly noteworthy, as well as the use of fluoroscopic and ultrasonic combinations for calculi localisation. Auxiliary measures have also improved the efficacy of ESWL and forced hydration as well as postural manoeuvers can facilitate the elimination of fragments. Otherwise, the use of a double $\mathrm{J}$ ureteral stent is often necessary for large stones. In cases of struvite calculi, the indications and modalities of irrigation of the urinary tract with litholytic solutions are still unclear. ${ }^{13,26-28}$

The potential long term effects on the renal parenchyma of the shock wave make the incidence of stone recurrence and the repeated use of ESWL treatment problematic and other modalities of the urological management of upper urinary tract calculi have a place.

Percutaneous nephrolithotomy has an 80 to $90 \%$ rate of success, but its early morbidity is particularly high among spinal cord injury patients. This technique is more efficient than ESWL when calculi measure more than $20 \mathrm{~mm}$ but frequent recurrences often counterbalance its initial advantages. Classical surgical measures allow successful removal of calculi and give a lower rate of recurrence, but the high morbidity rate in spinal cord damaged patients effectively limits its use to cases of staghorn calculi or stones associated with malformations of the urinary tract. ${ }^{10-13,29-37}$

Parallel to purely urological and technical advances, the medical treatment of calculi is of high importance and benefits from progress in pathophysiological discoveries concerning the lithogenic process. Prophylactic measures limit not only the frequency but also the rate of recurrence of calculi, and diminish their harmful effects on the upper urinary tract. ${ }^{4-10,13,38}$ Also, early detection of calculi allows for more successful treat- ment with ESWL by virtue of the smaller size of calculi encountered at the time of diagnosis.

\section{Conclusion}

ESWL is effective in spinal cord injury patients, but its particular usefulness and limitations in this patient population need to be kept in mind. Once fragmentation of the calculi is achieved, elimination from the urinary tract remains a problem. Its potential long term effects on the renal parenchyma dictate strict control of its repeated usage.

Thus, upper urinary tract calculi in spinal injury patients can benefit from this highly efficiency therapeutic measure, when used in a global context where prevention and early detection of calculi have to be given priority.

\section{References}

1 Banner M. Extracorporeal shock wave lithotripsy. Selection of patients and long-term complications. Radiol Clin North Am 1991; 29: 543-556.

2 Lingeman JE et al. The role of lithotripsy and its side effects. J Urol 1989; 141: 793-797.

3 Wilson WT, Preminger GM. Extracorporeal shock wave lithotripsy. An update. Urol Clin North Am 1990; 17: 207-216.

4 Devivo MJ, Fine PR, Cutter GR, Maetz HM. The risk of renal calculi in spinal cord injury patients. J Urol 1984; 131: 857-860.

5 Devivo MJ, Fine PR. Predicting renal calculus occurence in spinal cord injury patients. Arch Phys Med Rehabil 1986; 67: $722-725$.

6 Kohli A, Lamid S. Risk factors for renal stone formation in patients with spinal cord injury. Br J Urol 1986; 58: 588-591.

7 Nikakhtar B et al. Urolithiasis in patients with spinal cord injury. Paraplegia 1981; 19: 363-366.

8 Nuseibeh I, Burr RG. Survival time in paraplegic with certain urinary complications. Paraplegia 1982; 20: 270-276.

9 Stover SL, Lloyd LK, Waites KB, Jackson AM. Urinary tract infection in spinal cord injury. Arch Phys Med Rehabil 1989; 70: $47-54$

10 Gardner BP, Parsons KF, Soni BM, Krishnan KR. The management of upper urinary tract calculi in spinal cord damaged patients. Paraplegia 1985; 23: 371-378.

11 Irwin PP, Evans C, Chawla JC, Matthews PN. Stone surgery in the spinal patient. Paraplegia 1991; 29: 161-166.

12 Lazare JN, Saltzman B, Sotolongo J. Extracorporeal shock wave lithotripsy treatment of spinal cord injury patients. $J$ Urol 1988; 140: 266-269.

13 Levy DA, Resnick MI. Management of urinary stones in the patient with spinal cord injury. Urol Clin North Am 1993; 20: $435-442$.

14 Neuwirth H, Royce PL, Chaussy C. Use of extracorporeal shock wave lithotripsy in quadriplegic patients. JAMA 1986 ; 256: 1295.

15 Spirnak JP, Bodner D, Udayahankar S, Resnick M. Extracorporeal shock wave lithotripsy in traumatic quadriplegic patients: can it be safely performed without anesthesia? J Urol 1988; 139: 18-19.

16 Bierkens AF et al. Efficacy of second generation lithotriptors: a multicenter comparative study of 2206 extracorporeal shock wave lithotripsy treatments with the Siemens Lithostar, Dornier HM4, Wolff Piezolith 2300, Direx Tripter X-1 and Breakstone lithotriptors. J Urol 1992; 148: 1052-1057.

17 Bowsher WG et al. Clinical experience using the Wolff piezolith device at two British stone centers. J Urol 1989; 142: 679-682.

18 El Damanhoury $\mathrm{H}$ et al. Extracorporeal shock wave lithotripsy of urinary calculi: experience in treatment of 3278 patients using J Urol 1991; 145: 484-488.

19 Kim SC, Moon YT, Kim KD. Extracorporeal shock wave 
lithotripsy monotherapy: experience with piezoelectric second generation lithotriptor in 642 patients. J Urol 1989; 142: 674-678.

20 Lingeman JE et al. Extracorporeal shock wave lithotripsy: the Methodist Hospital of Indiana experience. J Urol 1986; 135: 1134-1137.

21 Marberger M, Turk C, Steinkogler I. Painless piezoelectric extracorporeal lithotripsy. J Urol 1988; 139: 695-699.

22 Simon J et al. Extracorporeal shock wave lithotripsy for urinary stone disease. Clinical experience with the electromagnetic lithotriptor 'Lithostar'. Eur Urol 1989; 16 7-11.

23 Beck EM, Riehle RA. The fate of residual fragments after extracorporeal shock wave lithotripsy monotherapy of infection stones. J Urol 1991; 145: 6-10.

24 Lerner SP, Gleeson MJ, Griffith D. Infection stones. J Urol 1989; 141: 753-758.

25 Michaels EK, Fowler JE. Extracorporeal shock wave lithotripsy for struvite renal calculi: prospective study with extended follow up. J Urol 1991; 146: 728-732.

26 Angermeier K, Streem SB, Yost A. Simplified infusion method for $10 \%$ hemiacidrin irrigation of renal pelvis. Urology 1993; 41: 243-246.

27 Brownlee N, Foster M, Griffith DP, Carlton CE Jr. Controlled inversion therapy: an adjunct to the elimination of gravitydependent fragments following extracorporeal shock wave lithotripsy. J Urol 1990; 143: 1096-1098.

28 Preminger GM et al. Ureteral stenting during extracorporeal shock wave lithotripsy: help or hindrance? J Urol 1989; 142 $32-36$.

29 Assimos DG et al. A comparison of anatrophic nephrolithotomy and percutaneous nephrolithotomy with and without extracorporeal shock wave lithotripsy for management of patients with staghorn calculi. J Urol 1991; 145: 710-714.

30 Chandhoke PS, Albala DM, Clayman RV. Long term comparison of renal function in patients with solitary kidneys and/or moderate renal insufficiency undergoing extracorporeal shock wave lithotripsy or percutaneous nephrolithotomy. J Urol 1992; 147: $1226-1230$

31 Coulange $\mathrm{C}$ et al. Scintigraphie rénale quantitative au DMSA après lithotritie extra-corporelle. Ann Urol 1990; 24: 322-325.

32 Lam HS et al. Staghorn calculi: analysis of treatment results between initial percutaneous nephrolithotomy and extracorporeal shock wave lithotripsy monotherapy with reference to surface area. J Urol 1992; 147: 1219-1225.

33 Mays N, Petruckevitch A, Burney PJ. Results of one and two year follow-up in a clinical comparison of extra-corporeal shock wave lithotripsy and percutaneous nephrolithotomy in the treatment of renal calculi. Scand J Urol Nephrol 1992; 26: 43-49.

34 Miller K, Bachor B, Sauter T, Hautmann R. Percutaneous nephrolithotomy/ESWL versus stent/ESWL for large stones and staghorn calculi: what we have learned? J Endourol 1989; 3: 287-293.

35 Roth RA, Beckmann CF. Complications of extracorporeal shock wave lithotripsy and percutaneous nephrolithotomy. Urol Clin North Am 1990; 17: 155-166.

36 Schulze $\mathrm{H}$ et al. Critical evaluation of treatment of staghorn calculi by percutaneous nephrolithotomy and extracorporeal shock wave lithotripsy. J Urol 1989; 141: 822-825.

37 Segura JW. Role of percutaneous procedures in the management of renal calculi. Urol Clin North Am 1990; 17: 207-216.

38 Burr RG, Nuseibeh I. Citrate excretion in spinal cord patients. Paraplegia 1990; 28: 496-504. 